\title{
An enhanced immune response against G250, induced by a heterologous DNA prime-protein boost vaccination, using polyethyleneimine as a DNA vaccine adjuvant
}

\author{
ZEQIANG SUN ${ }^{1}$, BO LIU ${ }^{2}$, XIYUN RUAN ${ }^{3}$ and QINGYONG LIU ${ }^{1}$ \\ ${ }^{1}$ Department of Urinary Surgery, Qianfoshan Hospital Affiliated to Shandong University, Jinan, Shangdong 250013; \\ ${ }^{2}$ Intensive Care Unit, Affiliated Hospital of Jinin Medical College, Jining, Shandong 272000; \\ ${ }^{3}$ Department of Neurology, Provincial Hospital Affiliated to Shandong University, Jinan, Shandong 250021, P.R. China
}

Received January 2, 2014; Accepted July 9, 2014

DOI: $10.3892 / \mathrm{mmr} .2014 .2537$

\begin{abstract}
The heterologous DNA prime-protein boost vaccination approach has been widely applied as an immune treatment for carcinoma. However, inefficient delivery of DNA remains a major issue. In the present study, polyethyleneimine (PEI) was used as a DNA vector carrier to improve the transfection efficiency of the DNA vaccine and stimulate the humoral and cellular immunity against the renal carcinoma-associated antigen G250. A protein vaccine was included in the immunization strategy in order to produce a prime-boost effect. A DNA plasmid encoding an antigen G250 fragment was constructed and complexed with PEI. A protein vaccine against G250 was expressed in BL21 (DE3) Escherichia coli cells, by transformation with a pET28a(+)/C-G250 plasmid. The protein was purified using a nickel-nitriloacetic acid purification system. The in vitro transfection efficiency of the DNA vaccine was analyzed in HEK293 human endothelial kidney cells. The in vitro transfection efficiency in HEK293 cells was highest $48 \mathrm{~h}$ after transfection. Furthermore, mice were primed with $200 \mu \mathrm{g}$ pVAX1/C-250 plasmid complexed with PEI, and boosted using $50 \mu \mathrm{g}$ of purified C-G250 protein. In order to evaluate the immune response the antibody titer, splenocyte response, and interferon- $\gamma$ levels from CD8+ T-cell splenocytes were analyzed using ELISA, lymphocyte proliferation or enzyme-linked immunosorbent spot assays. Firstly, the pVAX1/C-G250 plasmid was shown to be constructed successfully. As compared with the DNA group, the antibody titer, lymphocyte proliferation percentage, and cytokine production level induced by the DNA-PEI and DNA-PEI+C-G250 groups were significantly
\end{abstract}

Correspondence to: Dr Qingyong Liu, Department of Urinary Surgery, Qianfoshan Hospital Affiliated to Shandong University, 16766 Jingshi Road, Jinan, Shandong 250013, P.R. China

E-mail: lqylbc@163.com

Key words: renal cell carcinoma, G250, DNA vaccine, polyethyleneimine, prime-boost higher. Furthermore, the DNA-PEI+C-G250 group exhibited the strongest humoral and cellular response. Owing to the adjuvant effect of PEI, the pVAX1/C-G250-PEI prime plus $\mathrm{C}-\mathrm{G} 250$ protein boost regimen could induce a strong immune response, and has been proved to be a potent vaccine candidate against renal cell carcinoma.

\section{Introduction}

Renal cell carcinoma (RCC) is one of the most common urological conditions (1); however, due to high levels of resistance to chemotherapy and radiotherapy, the treatment of RCC is confounded by problems of tumor recurrence and metastasis (2). Since RCC is an immunogenic tumor, treatment with immunological therapies is possible. Numerous RCC patients have exhibited spontaneous, partial or complete remission, and immunotherapies have increased the reactivity of the immune system against RCC (3).

G250 protein, also known as carbonic anhydrase-9, is an isomer of the carbonic anhydrase enzyme family (4). The G250 gene can induce a malignant phenotype and is expressed in the majority of renal cell carcinomas, however it is not expressed in normal tissues $(3,5)$. The high expression levels of G250 in RCC makes G250 a potential target for immunotherapy (6). In previous studies, the RCC-associated antigen G250 was shown to encode naturally processed epitopes, which contain cytotoxic lymphocyte (CTL), and T-helper cell recognition sites. Both of these epitopes are found within the same region of the G250 protein; within amino acids 249-268 (7,8). However the immunogenicity of G250 is low because G250 produces a small, 20-kDa protein (9). In our previous study, the G250 gene was inserted into the major immunodominant region of the Hepatitis $\mathrm{B}$ core Antigen gene ( $\mathrm{HBcAg})$, resulting in the successful generation of the fusion protein C-G250 (10).

DNA vaccine constructs have been evaluated and tested in numerous human clinical trials for generating an immune response to various diseases, including infectious, allergic and autoimmune diseases, as well as cancers $(11,12)$. Unfortunately, the immunogenicity of plasmid DNA in humans has proven to be modest as compared with the immunogenicity observed 
in other species treated using microbial expression vectors. The synthetic polycationic polymer polyethylenimine (PEI), which consists of chains of ethylenimine units- $\mathrm{CH}_{2} \mathrm{CH}_{2} \mathrm{NH}-$, is considered to be the gold standard technique for enhancing the in vivo expression of administered genes $(13,14)$. PEI is superior to other non-microbial transfection agents because of its ability to protect DNA from degradation (15), to escape intracellular endosomal lysis, and to efficiently deliver DNA into the cell nucleus (16). Formulation with PEI polymers has been employed in DNA and siRNA (small interfering RNA)-based cancer immunotherapies (17) and in vaccination studies targeting infectious agents (18).

In the present study, pVAX1, a Food and Drug Administration-approved plasmid vector, was used as the carrier of G250 DNA, and combined with the synthetic PEI. The C-G250 protein was added to the immunization strategy in order to produce a prime-boost effect. The levels of humoral and cellular immune responses against G250 were observed to determine whether the DNA prime-G250 protein boost immunization strategy may provide a novel treatment strategy for RCC.

\section{Materials and methods}

Animals. BALB/c female mice, aged between 6 and 8 weeks, were housed in the Animal Experimental Center at the Medical College of Xi'an Jiaotong University (Xi'an, China). The mice received access to standard diet and water ad libitum and were housed under pathogen-free conditions in the Animal Experimental Center at the Medical College of Xi'an Jiatong University (Xi'an, China). All animal care and experimental procedures were approved by the Institutional Animal Care and Use Committee of the Xi'an Jiaotong University.

Construction and identification of the recombinant pVAX/C-G250 plasmid. The C-G250 gene was cloned from the $\mathrm{pET} 28 \mathrm{a}(+) / \mathrm{C}-\mathrm{G} 250$ plasmid and inserted into the eukaryotic expression vector pVAX1 (Invitrogen Life Technologies, Carlsbad, CA, USA) with EcoRI and XhoII restriction endonuclease sites. The recombinant plasmid pVAX1/C-G250 with the inserted sequence was verified by DNA sequencing. The plasmid was transformed into Escherichia coli Top10 competent cells (Invitrogen Life Technologies) for amplification purposes. Extraction and purification of the plasmid was performed using a DNA Extraction and Purification kit (Invitrogen Life Technologies). The recombinant plasmid was the transfected into HEK293 human embryonic kidney cells with Lipofectin ${ }^{\circledR}$ transfection reagent (Invitrogen Life Technologies), according to the manufacturer's instructions. The transfected cells were harvested and analyzed by reverse transcription-quantitative polymerase chain reaction (RT-qPCR) and western blotting, to determine the mRNA and protein C-G250 expression levels, respectively.

C-G250 expression and purification. The pET28a(+)/C-G250 plasmid was transformed into BL21 (DE3) E. coli cells and gene expression was induced with $1 \mathrm{mM}$ isopropyl $\beta$-D-1-thiogalactopyranoside. The expressed proteins were purified using a nickel-nitrilotriacetic acid (Ni-NTA) purification system (Invitrogen Life Technologies) and analyzed by
SDS-PAGE and western blotting. The bacterial lysates were resolved by Bis-Tris SDS-PAGE, and transferred to polyvinylidene fluoride (PVDF) membranes (Invitrolon ${ }^{\mathrm{TM}}, 0.45 \mathrm{~mm}$; Invitrogen Life Technologies). The western blots were probed with anti-G250 (American Research Products, Inc., Belmont, CA, USA) and anti-His antibodies (AbD Serotec, Raleigh, NC,USA) at 1:1,000 dilutions, followed by an incubation with alkaline phosphatase (AP)-conjugated rabbit antimouse immunoglobulin $\mathrm{G}(\mathrm{IgG})(\mathrm{H}+\mathrm{L})$ at a 1:2,500 dilution. AP detection was performed using a picoBLUE ${ }^{\mathrm{TM}}$ Immunoscreening kit (Stratagene, La Jolla, CA, USA).

$R T$ - $q P C R$. For qPCR, total RNA was extracted from transfected cells using TRIzol reagent (Invitrogen Life Technologies) according to manufacturer's instructions. Reverse transcription was performed on $2 \mu \mathrm{g}$ total RNA with the Superscript III First-Strand Synthesis System for RT-PCR kit (Invitrogen Life Technologies) using a mixture of oligo (dT)20 and random hexamer primers. The sequences of the primers for PCR were as follows: Sense: 5'-ATGATTACGCCAAGCTTGGG-3' and antisense: 5'-TCACGGAAGTGTTGATAGGA-3'. The PCR conditions were as follows: Denaturation at $94^{\circ} \mathrm{C}$ for $30 \mathrm{sec}$, annealing at $62^{\circ} \mathrm{C}$ for $30 \mathrm{sec}$, and extension at $72^{\circ} \mathrm{C}$ for $30 \mathrm{sec}$, which was repeated for 35 cycles. The PCR amplicons were then separated on $2.0 \%$ agarose gel by electrophoresis. The gel was stained with ethidium bromide, digitally photographed and scanned with the UVI Gel Analyzing system (UVI Tech, Cambridge, England).

Animal assay. BALB/c mice were divided into four groups $(n=5)$. The mice in the DNA group were injected with pVAX1/C-G250, the DNA-PEI complex group were injected with DNA-PEI complex at a ratio of 5:1. The injections were made into the quadriceps femoris muscles. The mice of the DNA and DNA-PEI groups were immunized four times over a 10-day period. The mice were immunized with $100 \mu \mathrm{l}$ of pVAX1/C-G250 $(2 \mu \mathrm{g} / \mu \mathrm{l})$ each. The following three immunizations, consisted of $50 \mu \mathrm{l}$ of pVAX1/C-G250. The mice of the DNA-PEI + protein group were immunized with $100 \mu \mathrm{l}$ and $50 \mu \mathrm{l}$ of DNA-PEI complex for the first two injections respectively, and then immunized with $50 \mu 1$ of DNA-PEI complex combined with $50 \mu \mathrm{g}$ of purified C-G250 protein for the final two injections. The blank group were injected with phosphate-buffered saline (PBS) at the same time points. Blood was collected 10 days after the last vaccination, incubated at $37^{\circ} \mathrm{C}$ for $30 \mathrm{~min}$, centrifuged at $800 \mathrm{x} \mathrm{g}$ for $10 \mathrm{~min}$, and then stored at $-80^{\circ} \mathrm{C}$, until further use.

ELISA to determine the antibodies raised against G250. 96-well plates were coated with purified C-G250 $(10 \mu \mathrm{g} / \mathrm{ml})$. The wells were blocked and $100 \mu$ l of test serum (pooled from the immunized mice), diluted at 1:100, was added to each well followed by a $1 \mathrm{~h}$ incubation at $37^{\circ} \mathrm{C}$. The bound antibody was probed with goat anti-mouse $\operatorname{IgG}(1: 2,000$, DAKO, Carpinteria, CA, USA), followed by an incubation with tetramethylbenzidine for 15-30 min. The optical density (OD) of the plates was read at $\mathrm{A} 450$.

Lymphocyte proliferation assay. Mice were sacrificed 10 days after the last immunization. Splenocytes were isolated from 
A

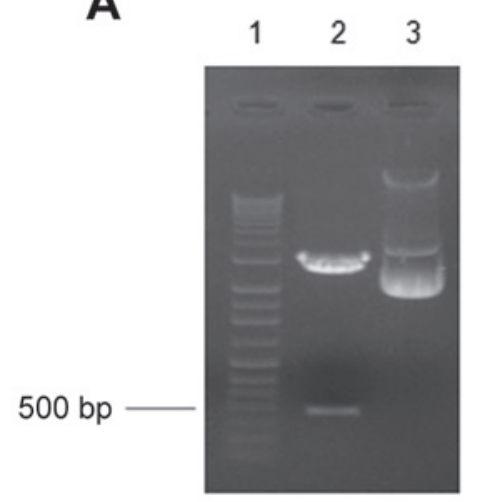

B

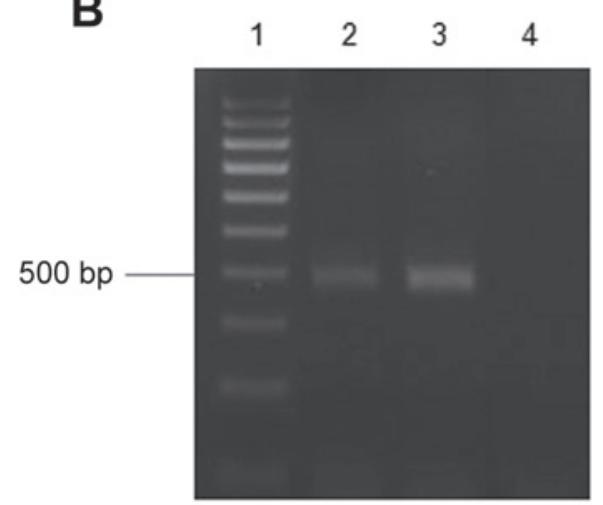

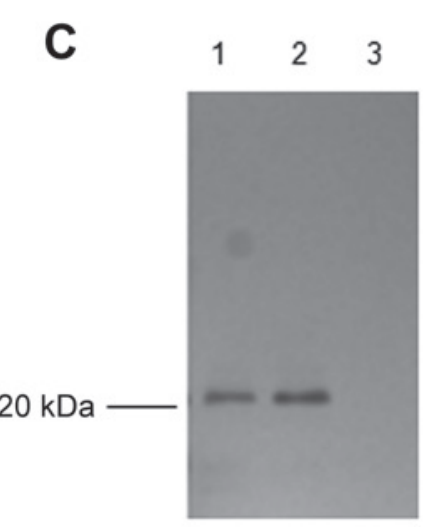

Figure 1. Construction and expression of C-G250 in human embryonic kidney (HEK) 293 cells. (A) The DNA band of C-G250 (508 bp) was observed $~ 500$ bp. Lane 1, DNA molecular marker; Lane 2, pVAX1/C-250 plasmid digested with EcoRI and XhoII. Lane 3, pVAX1/C-250 plasmid. (B) Quantitative polymerase chain reaction analysis of C-G250 expression in HEK293 cells. Lane 1, DNA molecular marker; Lane 2, cell lysates of HEK293 cells transfected with pVAX1/C-250 (24 h); Lane 3, polymerase chain reaction product of HEK293 cells transfected with pVAX1/C-250 (48 h); Lane 4, polymerase chain reaction product of HEK293 cells transfected with pVAX1 (48 h). (C) Western blot analysis of C-G250 expression in HEK293 cells. Lane 1, cell lysates of HEK293 cells transfected with pVAX1/C-250 (24 h); Lane 2, cell lysates of HEK293 cells transfected with pVAX1/C-G250 (48 h); Lane 3, cell lysates of HEK293 cells transfected with pVAX1 (48 h). Bp, base pairs; kDa, kilodaltons.

the mice and resuspended in RPMI-1640 medium supplemented with $10 \%$ fetal calf serum (Gibco-BRL, Carlsbad, CA, USA). A total of $2 \times 10^{4}$ splenocytes were seeded in triplicate in 96-well flat-bottom plates and incubated with $2 \mu \mathrm{g} / \mathrm{ml}$ purified C-G250 for 3 days. Following this, $0.5 \mathrm{mg} / \mathrm{ml} \mathrm{MTT}$ was added to the culture media and incubated at $37^{\circ} \mathrm{C}$ for $4 \mathrm{~h}$. Dimethyl sulfoxide was added to the culture media and the absorbance was then measured at A570. The splenic cell stimulation index (SI) was calculated by the formula: $\mathrm{SI}=$ stimulated group OD value/control group OD value.

Enzyme-linked immunosorbent spot (ELISPOT) analysis. Five days following the second of two weekly vaccinations, splenocytes were collected, depleted of red cells and CD8+ $\mathrm{T}$ cells were purified using the CD8a (Ly-2) Microbead kit (Miltenyi Biotech, Bergisch Gladbach, Germany) on an AutoMACS device (Miltenyi Biotech). ELISPOT analysis was performed to enumerate the number of CD8+ interferon- $\gamma$ (IFN- $\gamma$ )-producing cells, using the BD ${ }^{\mathrm{TM}}$ ELISPOT Mouse IFN- $\gamma$ kit (Becton Dickinson, Franklin Lakes, NJ, USA) and 96-well PVDF membrane plates (Millipore, Bedford, MA, USA), according to the manufacturer's instructions. Briefly, peritoneal exudate cells (PECs) were used as antigen-presenting cells; $1 \times 10^{5} \mathrm{PECs}$ from naïve BALB/c mice were placed in the ELISPOT wells, which were then loaded with $25 \mu \mathrm{g} / \mathrm{ml}$ of recombinant enhanced green fluorescent protein (EGFP) or $\mathrm{G} 250$ peptide in $100 \mu \mathrm{l}$ of media, for $4 \mathrm{~h}$ at $37^{\circ} \mathrm{C}$. CD8+ T cells $\left(1 \times 10^{5}\right.$ cells $\left./ 100 \mathrm{ml}\right)$ were added to each well for $18 \mathrm{~h}$ to test for antigen recognition. The spots were counted using an automated Immunospot 3 reader (Cellular Technology, Ltd., Cleveland, OH, USA).

Statistical analysis. The data were processed using SPSS version 17.0 for Windows (SPSS, Inc., Chicago, IL, USA). All the results are expressed as the means \pm standard deviation. One-way analysis of variance was employed to determine the differences amongst the groups. A $\mathrm{P}<0.05$ was considered to indicate a statistically significant difference.

\section{Results}

Construction and identification of the recombinant plasmids pVAX1/C-G250 expressing C-G250. The C-G250 gene was cloned and inserted into the eukaryotic expression vector pVAX. As shown in Fig. 1A, a band was present at $200 \mathrm{bp}$, when the recombinant $\mathrm{pVAX} 1 / \mathrm{C}-\mathrm{G} 250$ was digested with EcoRI and XhoI. The DNA sequencing data confirmed that the $\mathrm{C}-\mathrm{G} 250$ gene had been cloned into the pVAX1 expression vector successfully (data not shown). The qPCR and western blotting data showed that C-G250 was expressed in the HEK293 cells which were transfected with pVAX1/C-G250 at a concentration of $2 \mu \mathrm{g} / \mathrm{ml}$. The bands of the qPCR and western blot analysis (Fig. 1B and C) identified that C-G250 expression $48 \mathrm{~h}$ after transfection was increased as compared with the expression levels $24 \mathrm{~h}$ after transfection.

C-G250 expression and purification from pET28a(+)/C-G250. Recombinant C-G250 was expressed in the pET28a bacterial vector, encoding an N-terminal polyhistidine-tag (His6) sequence. The proteins containing the His6 tag were purified from the bacterial lysates using a Ni-NTA column. SDS-PAGE analysis detected a new band $\sim 20 \mathrm{kDa}$ produced in bacteria that were transformed with pET28a(+)/C-G250 (Fig. 2A and B). The purified protein was also $\sim 20 \mathrm{kDa}$ and was recognized by both the anti-His and anti-G250 antibodies (Fig. 2C).

G250-specific antibodies are detected in C-G250-immunized mice. BALB/c mice were divided into four groups: Blank, DNA, DNA-PEI and DNA-PEI + protein groups. The serum IgG levels of the mice from the groups was determined by ELISA. As shown in Fig. 3, G250-specific IgG antibodies were detected in the sera of the DNA, DNA-PEI and DNA-PEI + protein groups following the final immunizations; however, they were not detected in the blank group.

The IgG level of the DNA-PEI group was significantly higher as compared with the DNA group. The highest antibody titer was found when the DNA-PEI complex + C-G250 protein was 

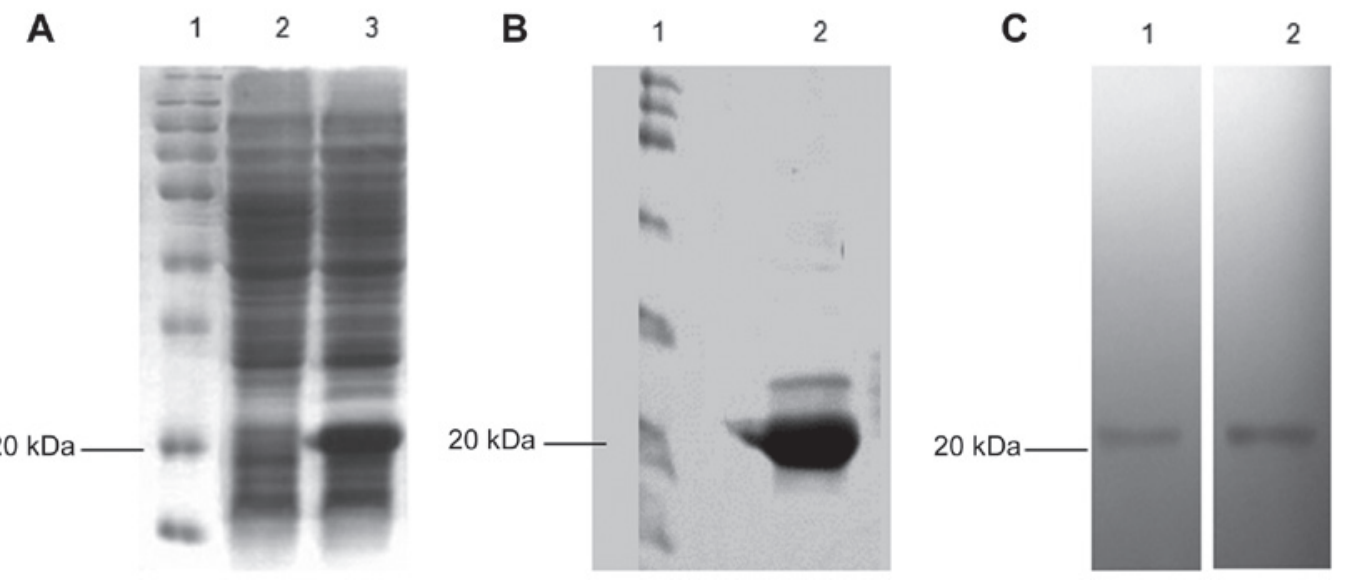

Figure 2. Expression and purification of C-G250. (A) C-G250 was expressed by the pET28a(+) bacterial vector in BL21 (DE3) Escherichia coli cells. A distinct band of $20 \mathrm{kDa}$, corresponding to C-G250, was resolved by SDS-PAGE. Lane 1, protein molecular marker; Lane 2, bacteria transformed with empty pET28a(+) vector; Lane 3, bacteria transformed with C-G250 recombinant pET28a(+) vector. (B) Recombinant C-G250 purified with a nickel-nitriloacetic acid column. Lane 1, protein molecular marker; Lane 2, purified protein from bacteria transformed with C-G250 recombinant pET28a(+) vector. (C) Western blot analysis of the purified C-G250. Lane 1, proteins probed with anti-His antibody; Lane 2, proteins probed with anti-G250 antibody. kDa, kilodaltons.

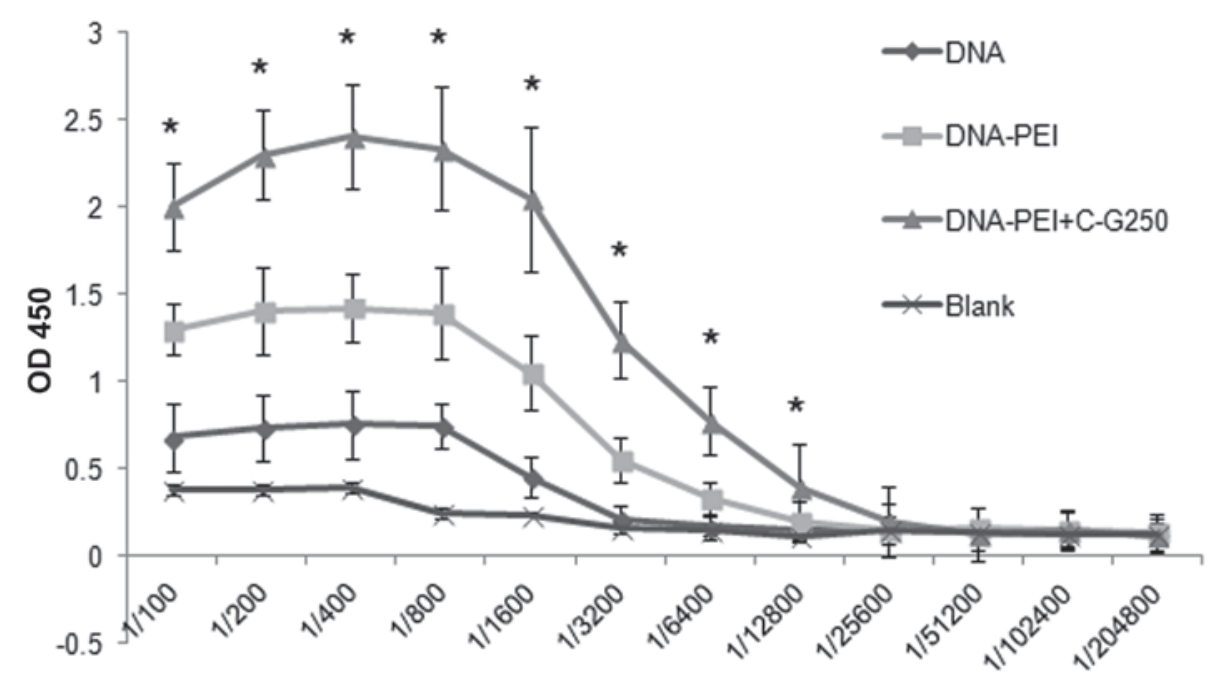

Figure 3. Specific immunoglobulin G levels of C-G250 in immunized mice. DNA group, mice immunized with pVAX1/C-G250 only; DNA-PEI group, mice immunized with pVAX1/C-G250 and PEI complex; DNA-PEI+C-G250 group, mice immunized with pVAX1/C-G250 and PEI complex for the first two times and immunized with DNA-PEI complex combined with C-G250 for the final two times; Blank group, mice immunized with phosphate-buffered saline. The error bars represent the standard deviations for triplicate sample wells in the same assay. ${ }^{*}<0.05$ vs. the blank group. OD, optical density; PEI, polyethyleneimine.

A

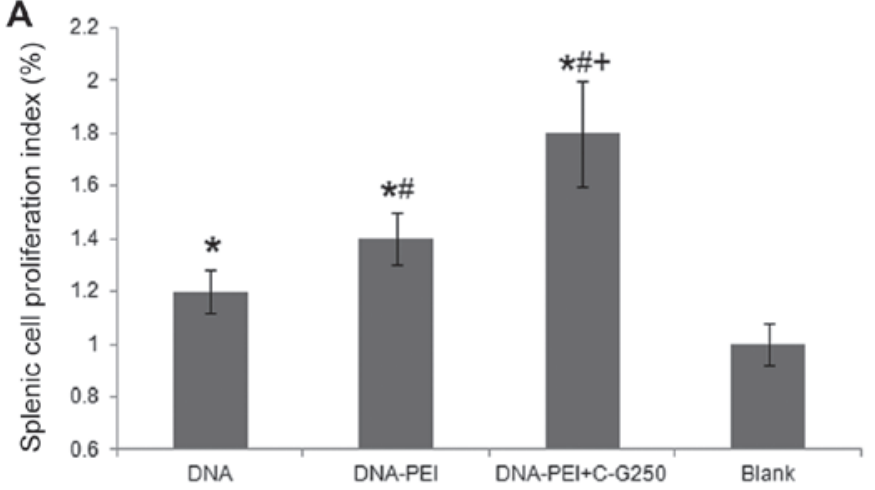

B

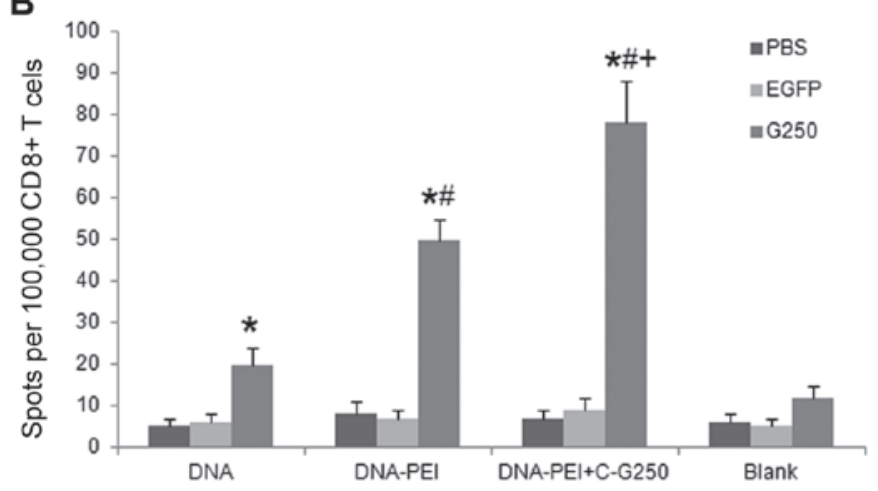

Figure 4. Antigen-specific responses of splenocytes. (A) G250-induced antigen specific proliferative assay of splenocytes. (B) IFN- $\gamma$ ELISPOT assay of CD8+ T-cell response to G250. PBS group, peritoneal exudate cells (PEC) loaded with phosphate-buffered saline; EGFP group, PEC loaded with enhanced green fluorescent protein; G250 group; PEC loaded with G250 peptide. The error bars represent standard deviations for triplicate sample wells in the same assay. ${ }^{*} \mathrm{P}<0.05$ vs. blank group, ${ }^{*} \mathrm{P}<0.05$ vs. DNA group, ${ }^{+} \mathrm{P}<0.05$ vs. DNA-PEI group. PEI, polyethyleneimine. 
used to immunize the mice. This difference was significant at the 1:6,400 dilution as compared with the other groups (Fig. 3).

Antigen-specific responses of splenocytes. Ten days after the final immunization, splenocytes were isolated and stimulated with G250 peptide to analyze the cell proliferation. As shown in Fig. 4A, splenocytes from all of the immunized groups responded to G250 stimulation, and the SI of the DNA-PEI+C-G250 group was significantly higher as compared with the DNA and DNA-PEI groups $(\mathrm{P}<0.05)$. CD8+ T-cell response against G250 was also detected using an IFN- $\gamma$ ELISPOT assay, in which PECs were used as the antigen presenting cells. CD8 $+\mathrm{T}$ cells were enriched from each group by immune magnetic selection. Control cultures, containing either T cells plus PBS or EGFP-loaded PECs, had low numbers of IFN- $\gamma$ producing cells. In contrast, significant anti-G250 reactivity was found in $\mathrm{T}$ cells of all the immunized groups, with the highest anti-G250 reactivity in the DNA-PEI + C-G250 group (Fig. 4B).

\section{Discussion}

RCC belongs to a small group of immunogenic tumors. In patients with RCC, partial or complete remission has been observed in response to immunotherapy, and continued immunotherapy treatment can increase the immune reaction against RCC (3). The presence of CD4+ T-lymphocytes and CD8+ T-lymphocytes in RCC supports the hypothesis that the immune system is triggered in these tumors (7). Only a small number of RCC-associated antigens have been characterized that are recognized by CD8+ CTLs, including advanced glycosylation end product-specific receptor, receptor tyrosine-protein kinase erbB-2, G250 and squamous cell carcinoma antigen recognized by T-cells $3(8,19-20)$.

The present study aimed at improving the efficacy of the heterologous DNA prime-protein boost vaccination strategy against the RCC-associated antigen G250, in inducing both cellular and humoral immune responses. Previous research has demonstrated the capability of a DNA prime-protein boost regimen, which can elicit both an antigen-specific antibody and cytotoxic T-cell response (22-24). Problems, including the low transfection efficiency of the DNA vector, and the low immunogenicity of the antigens, remain to be overcome. In the present study, low molecular weight PEI was used as a DNA vector carrier in order to enhance the transfection efficiency, and the G250 gene was fused with the major immunodominant region (MIR) of the HBcAg gene (C-G250) to increase the immunogenicity of the G250 antigen. Following boosting the vaccine with the fusion protein $\mathrm{C}-\mathrm{G} 250$, both the $\mathrm{CD} 8+\mathrm{T}$-cell response and the CD4+ T-cell response were significantly enhanced.

PEI has been previously used in gene therapy since its initial introduction as a gene delivery vehicle (13). The PEI/DNA complex can prolong DNA retention, and enhance the transfection of the target gene $(25,26)$. Furthermore, PEI has previously been used in a heterologous prime-boost vaccination strategy (13). Li et al (22) explored a heterologous approach using DNA prime-adenovirus boost vaccination with PEI as a DNA vaccine adjuvant. The results indicated that PEI could be used as a competent carrier for the delivery of a DNA vaccine. This idea was supported by Wegmann et al (27), who showed that PEI was a safe and efficient DNA vaccine adjuvant that induced a stronger immune response.

In the present study, PEI was used as a DNA carrier in order to improve and retain a high transfection efficiency. The DNA-PEI complex group demonstrated an improved T-cell immune response and higher antibody levels as compared with the DNA group. These results indicate that PEI has a strong adjuvant effect. Thus, it can be inferred from these results that PEI is a potent DNA vaccine carrier, which can induce a strong immune response.

The boost vaccination of C-G250 significantly enhanced the immune responses as compared with the DNA vaccination alone. In the DNA-PEI+protein group, antigen-specific antibody $\mathrm{IgG}$, and antigen-specific CTL levels were measured. From the results, it can be observed that following protein boosting, the IgG titer of the DNA-PEI+protein group was significantly higher as compared with the DNA and DNA-PEI groups (Fig. 3). A significant anti-G250 reactivity was found in CD8+ T cells of all of the immunized groups, especially in the DNA-PEI+C-G250 group, indicating that the cellular and humoral immune responses, were enhanced by the DNA prime-protein boost regimen.

In conclusion, PEI was used as a DNA vaccine carrier and the effects of a heterologous DNA priming plus protein boosting vaccination strategy were evaluated in mice. The results indicated that the DNA-PEI+protein regimen significantly enhanced the anti-G250 immune response, as compared with the DNA and DNA-PEI groups. This resulted in higher CD8+ and CD4+ T-cell responses and cytokine production levels, which make this strategy a potential vaccine candidate against RCC. Future research may focus on the mechanism of vaccination, and evaluate its efficacy on an animal model of RCC.

\section{Acknowledgements}

This study was supported by grants from the Technology Research Project of Shaanxi Province (nos. 2012SF2-21 and 2012K16-09-02), and the National Natural Science Foundation of China (nos. 81200545 and 81270545).

\section{References}

1. Awakura Y, Ito N, Nakamura E, et al: Matrix metalloproteinase-9 polymorphisms and renal cell carcinoma in a Japanese population. Cancer Lett 241: 59-63, 2006.

2. Dutcher JP: Recent developments in the treatment of renal cell carcinoma. Ther Adv Urol 5: 338-353, 2013.

3. Tostain J,Li G, Gentil-Perret A and Gigante M: Carbonic anhydrase 9 in clear cell renal cell carcinoma: a marker for diagnosis, prognosis and treatment. Eur J Cancer 46: 3141-3148, 2010.

4. Stadick H, Stockmeyer B, Kühn R, et al: Epidermal growth factor receptor and g250: useful target antigens for antibody mediated cellular cytotoxicity against renal cell carcinoma? J Urol 167: 707-712, 2002.

5. Li G, Feng G, Gentil-Perret A, Genin C and Tostain J: CA9 gene expression in conventional renal cell carcinoma: a potential marker for prediction of early metastasis after nephrectomy. Clin Exp Metastasis 24: 149-155, 2007.

6. Muselaers S, Mulders P, Oosterwijk E, Oyen W and Boerman O: Molecular imaging and carbonic anhydrase IX-targeted radioimmunotherapy in clear cell renal cell carcinoma. Immunotherapy 5: 489-495, 2013.

7. Vissers JL, De Vries IJ, Engelen LP, et al: Renal cell carcinoma-associated antigen $\mathrm{G} 250$ encodes a naturally processed epitope presented by human leukocyte antigen-DR molecules to CD4(+) T lymphocytes. Int J Cancer 100: 441-444, 2002. 
8. Vissers JL, De Vries IJ, Schreurs MW, et al: The renal cell carcinoma-associated antigen G250 encodes a human leukocyte antigen (HLA)-A2.1-restricted epitope recognized by cytotoxic T lymphocytes. Cancer Res 59: 5554-5559, 1999.

9. Xiao Y, Gao J, Gao K, et al: Prokaryotic expression, purification and antigenicity identification of human renal cell carcinoma-associated antigen G250. Xi Bao Yu Fen Zi Mian Yi Xue Za Zhi 29: 269-272, 2013. (In Chinese).

10. Liu B,Sun Z, Liu Q, et al: Construction and prokaryotic expression of recombinant gene G250 antigenic peptide-HBcAg and the immunogenicity analysis of the fusion protein. Journal of Xi'an Jiaotong University (Medical Sciences) 1: 6-11, 2014.

11. Manoj S, Babiuk LA and van Drunen Littel-van den Hurk S: Approaches to enhance the efficacy of DNA vaccines. Crit Rev Clin Lab Sci 41: 1-39, 2004.

12. Ada G: Vaccines and vaccination. N Engl J Med 345: 1042-1053, 2001.

13. Grant EV, Thomas M, Fortune J, Klibanov AM and Letvin NL: Enhancement of plasmid DNA immunogenicity with linear polyethylenimine. Eur J Immunol 42: 2937-2948, 2012.

14. Krishnamachari Y, Geary SM, Lemke CD and Salem AK: Nanoparticle delivery systems in cancer vaccines. Pharm Res 28: 215-236, 2011

15. Demeneix B and Behr JP: Polyethylenimine (PEI). Adv Genet 53PA: 215-230, 2005.

16. Kichler A, Leborgne C, Coeytaux E and Danos O: Polyethylenimine-mediated gene delivery: a mechanistic study. J Gene Med 3: 135-144, 2001.

17. Ma YF and Yang YW: Delivery of DNA-based cancer vaccine with polyethylenimine. Eur J Pharm Sci 40: 75-83, 2010.

18. Bivas-Benita M, Bar L, Gillard GO, et al: Efficient generation of mucosal and systemic antigen-specific CD8+ T-cell responses following pulmonary DNA immunization. J Virol 84: 5764-5774, 2010 .
19. Gaugler B, Brouwenstijn N, Vantomme V, et al: A new gene coding for an antigen recognized by autologous cytolytic $\mathrm{T}$ lymphocytes on a human renal carcinoma. Immunogenetics 44 . 323-330, 1996

20. Brossart P, Stuhler G, Flad T, et al: Her-2/neu-derived peptides are tumor-associated antigens expressed by human renal cell and colon carcinoma lines and are recognized by in vitro induced specific cytotoxic T lymphocytes. Cancer Res 58: 732-736, 1998

21. Kawagoe N, Shintaku I, Yutani S, et al: Expression of the SART3 tumor rejection antigen in renal cell carcinoma. J Urol 164: 2090-2095, 2000.

22. Li K, Gao H, Gao L, et al: Enhancement of humoral and cellular immunity in chickens against reticuloendotheliosis virus by DNA prime-protein boost vaccination. Vaccine 31: 1944-1949, 2013.

23. Li M, Jiang Y, Xu C, Zhang Z and Sun X: Enhanced immune response against HIV-1 induced by a heterologous DNA prime-adenovirus boost vaccination using mannosylated polyethyleneimine as DNA vaccine adjuvant. Int J Nanomedicine 8: $1843-1854,2013$

24. Lan J, Gao Z, Xiong H, et al: Generation of protective immune responses against coxsackievirus B3 challenge by DNA prime-protein boost vaccination. Vaccine 29: 6894-6902, 2011.

25. Huang X, Xu J, Qiu C, et al: Mucosal priming with PEI/DNA complex and systemic boosting with recombinant TianTan vaccinia stimulate vigorous mucosal and systemic immune responses. Vaccine 25: 2620-2629, 2007.

26. Goula D, Becker N, Lemkine GF, et al: Rapid crossing of the pulmonary endothelial barrier by polyethylenimine/DNA complexes. Gene Ther 7: 499-504, 2000.

27. Wegmann F, Gartlan KH, Harandi AM, et al: Polyethyleneimine is a potent mucosal adjuvant for viral glycoprotein antigens. Nat Biotechnol 30: 883-888, 2012. 